\title{
Clumping of lymphoma cells in peripheral blood induced by EDTA
}

\author{
S Juneja, $M$ Wolf, R McLennan
}

\begin{abstract}
A peripheral blood smear from a patient with probable splenic lymphoma with villous lymphocytes (SLVL) showed clumping of lymphoma cells. The clumping was not seen in films made from unanticoagulated blood, and has not been previously described in lymphomas. The patient also had metastatic prostatic adenocarcinoma for $\mathbf{3 0}$ months before lymphoma was diagnosed and the clumped cells posed diagnostic problems.
\end{abstract}

Evidence of lymphoma in peripheral blood at presentation is uncommon and the incidence varies according to histological subtype. ${ }^{1} \mathrm{Cir}-$ culating tumour cells of non-haematological malignancies are rarely found when peripheral blood is examined. ${ }^{2}$ When present, the lymphoma cells are seen singly whereas the carcinoma cells tend to occur in clumps. ${ }^{2}$ We describe a patient with a known adenocarcinoma for 30 months who posed diagnostic problems because he presented at one stage with clumps of tumour cells in the peripheral blood. These turned out to be the cells of an unusual type of lymphoma showing clumping induced by EDTA.

\section{Methods}

The blood was collected in $\mathrm{K}_{2}$ EDTA and a full blood examination (FBE) was performed with the Coulter S-Plus IV and the differential count was done manually. Immunological marker studies were carried out on mononuclear cell suspensions separated from blood and bone marrow on Ficoll/hypaque. Cells were washed twice in phosphate buffered saline and stained for surface immunoglobulin using $F\left(a b^{\prime}\right)$ fragments of goat anti-human Ig sera conjugated to fluorescein isothiocyanate (FITC), polyvalent and $\gamma, \mu, \alpha, \delta, \kappa$ and $\lambda$ specific antisera. Sheep erythrocyte, mouse erythrocyte antibody rosette tests were performed according to previously described methods. ${ }^{3}$ The bone marrow biopsy specimen was stained with monoclonal antibodies using the four-layer immunoperoxidase (PAP) technique.

\section{Case report}

A 66 year old man presented in October 1987 with a lytic lesion in the right lower tibia. Bone scans showed additional hot spots in the right humeral shaft, the transverse process of the fifth thoracic vertebra and ischium and an extensive periosteal reaction in the right lower tibia. A biopsy specimen of the right tibial osteolytic lesion showed a well differentiated adenocarcinoma. The tumour was negative for prostate specific stains. Rectal examination showed some irregularity in the right lobe of the prostate which was not thought to be malignant. A prostatic biopsy specimen contained benign tissue only and a limited transurethral resection of the prostate was carried out. Investigation of the gastrointestinal tract and lung did not indicate the presence of any primary tumour. Metastatic adenocarcinoma of unknown primary site was diagnosed.

He was treated with a course of radiotherapy to the right shoulder and right lower leg in December 1987, to the face and neck in February 1988, lumbar spine in May 1988, thoracic and lumbar vertebrae in July 1988, and pelvis in October 1988. In 1989 he received six courses of CMF (cyclophosphamide, methotrexate, and 5-fluorouracil) chemotherapy for two cycles, with no response. Further chemotherapy with vincristine and mitoxantrone, followed by radiotherapy to the right leg, was given for relief of pain. In March 1990 he had a pathological fracture of right lower ribs that required local radiotherapy. One month later he was admitted with severe pain in the left foot and bilateral L4 and L5 nerve root pain. A myelogram showed cauda equina syndrome and he was admitted for urgent radiotherapy.

On physical examination he had a soft mobile submandibular lymph node but no other palpable lymphadenopathy. The spleen and liver were enlarged. There was tenderness over the lower lumbar and sacroiliac joints. Investigations showed the serum calcium to be $2 \cdot 11$ $\mathrm{mmol} / \mathrm{l} \quad(2 \cdot 10-2 \cdot 60)$, bilirubin $14 \mathrm{mmol} / 1$ (0-17), alkaline phosphatase $584 \mathrm{IU} / 1(30-120)$, gammaGT $69 \mathrm{IU} / 1$ (15-162), aspartate transaminase $17 \mathrm{IU} / 1(5-40)$, total protein $62 \mathrm{~g} / 1$ (60-80), and albumin $24 \mathrm{~g} / 1$ (34-47). A full blood picture showed that haemoglobin was $119 \mathrm{~g} / \mathrm{l}$, the white cell count was $8.1 \times 10^{9} / 1$ and platelets were $209 \times 10^{9} / 1$. A differential count showed that $30 \%$ were abnormal cells which were present singly and in clumps of 4-50 cells (fig 1): these cells were $10-15 \mu \mathrm{m}$ in diameter with moderate nuclear:cytoplasmic ratio. The nuclei were rounded in most cells with occasional irregular forms. The nuclear chromatin was relatively condensed but was finer than that seen in cells of chronic lymphocytic leukaemia. There were occasional lymphoplasmacytic cells but this was not a striking feature. The cytoplasm was pale blue and in many cells showed hairy projections which were often seen at one pole rather than 
Figure 1 Peripheral blood smear showing a clump of lymphoma cells (Inset individual lymphoma cell).

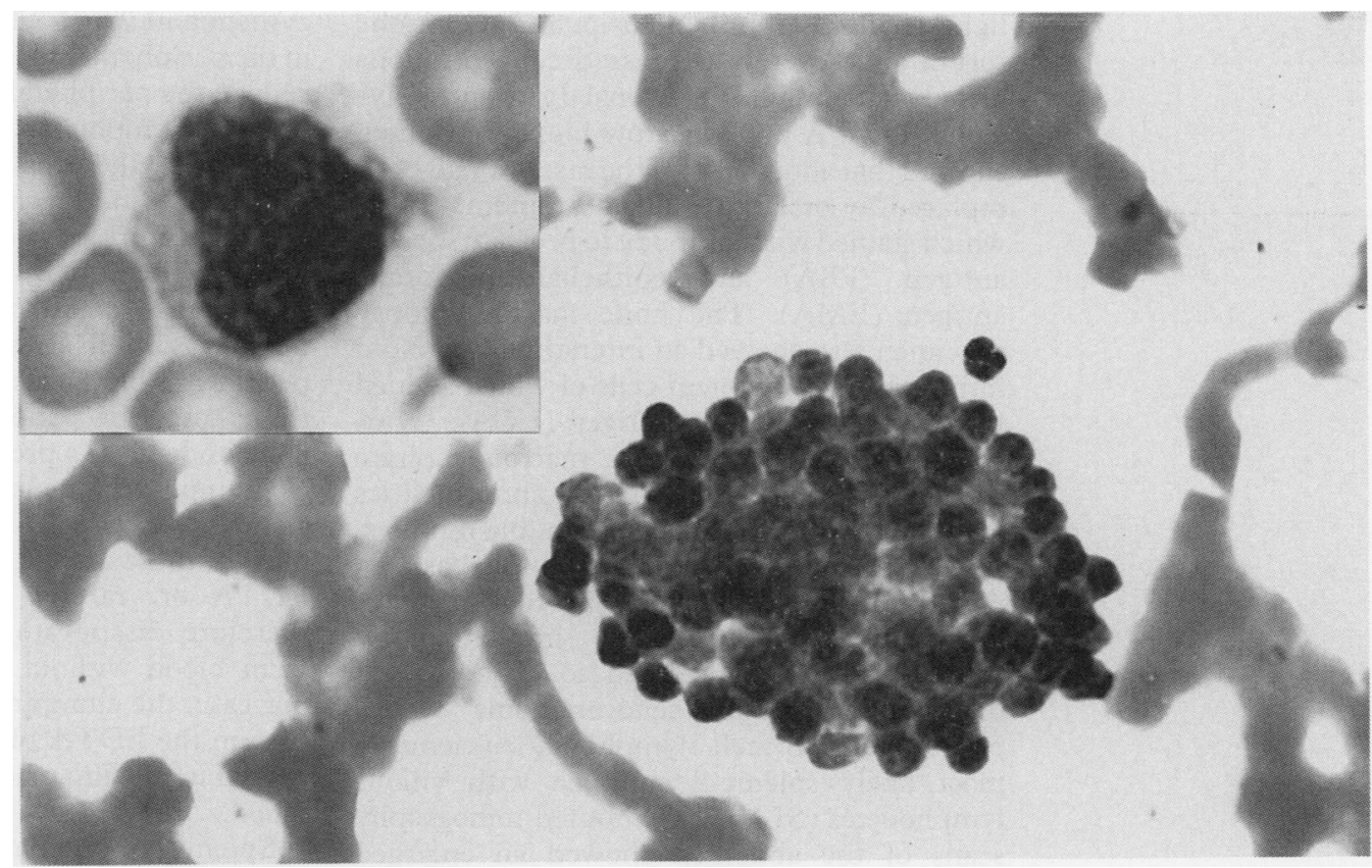

being distributed uniformly all around the cell surface as in hairy cell leukaemia (fig 1 ; inset). The tumour cells did not stain with acid phosphatase or periodic acid Schiff (PAS) stains. Clumping of tumour cells was also seen in repeat blood specimens collected in EDTA but was absent in smears made from direct venepuncture specimens without the added anticoagulant. Immunological marker studies on cell suspensions showed that the tumour cells were monoclonal $(\mu, \delta$, and $\lambda)$. The surface

immunoglobulin was bright and only $1 \%$ cells formed mouse rosettes. The lymphoid cells stained with Ia, CD5, 11a, 20, 22, 32B, 33, 37, $38,40, F M C 1$ and FMC7. CD11C stained only $3 \%$ of the cells. Staining with monoclonal antibodies HC2 and CD25 was not carried out.

The immunological markers were confirmed on immunoperoxidase staining of the blood smears where the tumour cell clumps stained positively with the above B cell markers. There were low concentrations of monoclonal free
Figure 2 Bone marrow biopsy specimen showing metastatic carcinoma.

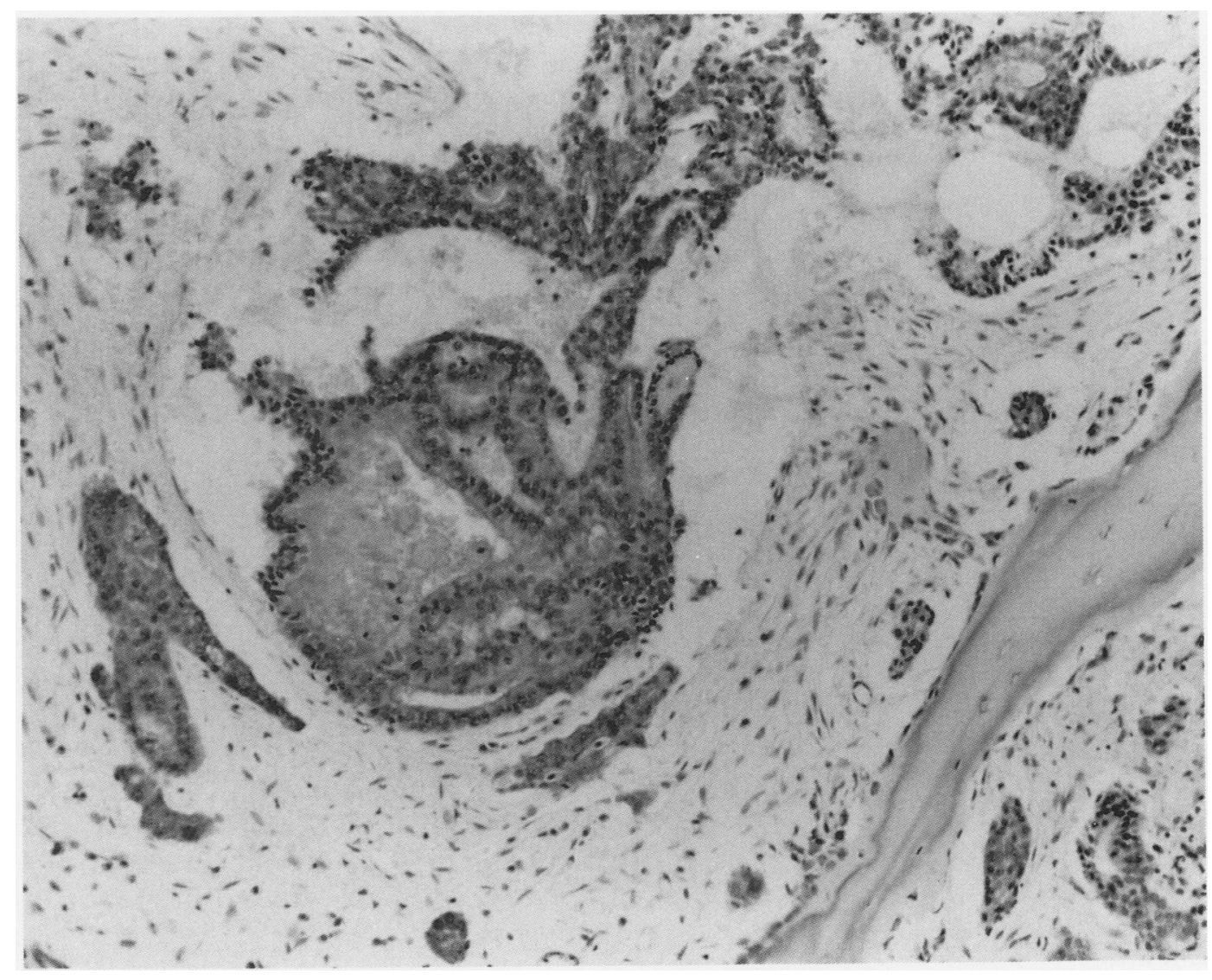


light chains $(\lambda)$ and oligoclonal IgG in the serum and severely depressed concentrations of polyclonal IgG, polyclonal IgA, and polyclonal IgM. A bone marrow biopsy specimen showed that about $50 \%$ of the marrow had been replaced by metastatic adenocarcinoma (fig 2) which stained with antibody to prostate specific antigen (PSA) and epithelial membrane antigen (EMA). The bone marrow biopsy specimen also showed an interstitial lymphoid infiltrate the component cells of which stained for leucocyte common antigen (LCA). Cytogenetic studies on the bone marrow aspirate showed multiple and complex abnormalities including markers, rings, and double minutes, consistent with carcinoma. On the basis of peripheral blood and bone marrow morphology, cytochemistry, and immunological features, it was considered to be a case of metastatic prostatic adenocarcinoma with a concomitant B cell lymphoma/leukaemiamost likely splenic lymphoma with villous lymphocytes (SLVL). Computed tomography scans of the abdomen showed an enlarged spleen. The patient was started on a course of Zoladex (Goserelin) for treatment of prostate cancer. Over the next few weeks the spleen became palpable below the costal margin with a rising white cell count. He was given combination chemotherapy consisting of vincristine, prednisolone, and mitoxantrone. He achieved a partial response $(50 \%$ or more reduction in the circulating lymphoma cells or reduction in spleen size). Sepsis developed after the second course of the above combination chemotherapy at which stage he abandoned treatment and subsequently died in October 1990.

\section{Discussion}

To our knowledge this is the first case of lymphoma in which the circulating lymphoma cells in the peripheral blood showed EDTA induced clumping. Moreover, this was a known case of metastatic adenocarcinoma, thus leading to diagnostic confusion. In our experience of several hundred lymphoma cases at our institution we have never seen clumping of lymphoma cells in the peripheral blood. Cell clumping is a characteristic feature of metastatic carcinoma in bone marrow aspirates and has been reported in very rare cases of carcino- cythemia in which carcinoma cells are present in the peripheral blood. ${ }^{2}$ In our case the tumour cells in the peripheral blood were clearly identified as lymphoma cells and not carcinoma cells. First, the cells marked as monoclonal B cells and did not mark with antibodies to epithelial antigens. Second, there was a simultaneous interstitial infiltration of the marrow by identical small mononuclear cells which marked as B cells. Lastly, carcinoma cells in the peripheral blood are extremely rare and in these cases the patients usually present with pancytopenia as a preterminal event.

Clumping of neutrophils ${ }^{4}$ and platelets ${ }^{5}$ is sometimes seen in the peripheral blood and in most of these cases it occurs in the presence of EDTA or, rarely, other anticoagulants. It therefore disappears when the film is made from blood without added anticoagulant. In our case, the clumping of lymphoma cells was seen in the EDTA blood specimen and disappeared in the film made from unanticoagulated blood.

Splenic lymphoma with villous lymphocytes (SLVL) had been recently described. ${ }^{6}$ These cases occur in middle aged to elderly persons, more commonly in men, and they usually have massive splenic enlargement. The peripheral blood shows lymphocytes with villous projections which are often localised to one pole of the cell. The cells are monoclonal B lymphocytes but differ from hairy cell leukaemia in that they do not react with $\mathrm{HC} 2, \mathrm{CD} 25$, Leu M5 or cytochemically with tartrate resistant acid phosphatase. Our case fulfilled many of the above criteria.

1 Juneja SK, Wolf M, Cooper IA. Value of bilateral biopsies in non-Hodgkin's lymphoma. J Clin Pathol 1990;43:630-2. 2 Gallivan MVE, Libich JJ. Carcinocythemia (carcinoma cell leukaemia). Cancer 1984;53:1100-2.

3 Jose DG, Pilkington GR, Wolf MM, et al. Diagnostic information derived from immunophenotyping 1000 patients with leukaemia and lymphoma. Pathology 1983; 15:53-60.

4 Epstein HD, Kruskall MS. Spurious leukopenia due to in vitro granulocyte aggregation. Am J Clin Pathol 1988:89: $652-5$

5 George JN, Aster RH. Quantitative platelet disorders. In: William WJ, Butler E, Erslev RJ, Lichtman MA, eds. Hematology. 4th Edn. London: McGraw-Hill, 1990:1343. 6 Melo JV, Hegde V, Parreira A, et al. Splenic B cell lymphoma with circulating villous lymphocytes: differential diagnosis of $\mathbf{B}$ cell leukaemias with large spleens. J Clin Pathol 1987;40:642-51. 